\title{
A 3D Matching Method for Organic Training Samples Alignment Based on Surface Curvature Distribution
}

\author{
Guangxu Li, Hyoungseop Kim, Joo Kooi Tan, Seiji Ishikawa, Akiyoshi Yamamoto \\ Kyushu Institute of Technology, Kitakyushu, Japan \\ E-mail:kim@cntl.kyutech.ac.jp \\ Received October 19, 2011; revised November 18, 2011; accepted November 28, 2011
}

\begin{abstract}
The fundamental step to get a Statistical Shape Model (SSM) is to align all the training samples to the same spatial modality. In this paper, we propose a new 3D alignment method for organic training samples matching, whose modalities are orientable and surface figures could be recognized. It is a feature based alignment method which matches two models depending on the distribution of surface curvature. According to the affine transformation on 2D Gaussian map, the distances between the corresponding parts on surface could be minimized. We applied our proposed method on 5 cases left lung training samples alignment and 4 cases liver training samples alignment. The experiment results were performed on the left lung training samples and the liver training samples. The availability of proposed method was confirmed.
\end{abstract}

Keywords: Training Samples Alignment, Statistical Shape Model, Gauss Map, K-Means

\section{Introduction}

Due to utilizing the priori information of shapes, Statistical Shape Model (SSM) method shows concise and robust for segmentation, analyzing and interpreting anatomical objects from medical datasets [1]. The basic idea in model building is to statistic the pattern of legal variation in the shapes and spatial relationships of structures from a collection of training samples. A key step in building a model involves establishing a dense correspondence between shape boundaries and surface figures. It is important to align all training samples in a common coordinate frame firstly [2].

The training shapes alignment problems can be concluded that the transformation between the same anatomy images at different modalities or represented by one modality at different time [3]. In point sets based alignment method, the set of identified points is sparse compared with the original image content, which makes for relatively fast optimization procedures, but is difficult to confirm the corresponding points. Some novel strategies are reported to optimize measures such as the average distance between each representative point, or iterated minimal distances metric [4]. These methods are mostly used to find rigid or affine transformations. Featurebased alignment is largely founded on the use of differential geometry to describe local surface feature. Ac- cording to the difference of local feature on parameterized space, an appropriate transformation could be required. The statistical model building would benefit from this technology. Because generally the landmarks positions always involve the local features of surface.

In this paper, we propose a feature-based alignment method to reconcile the training samples of organs field whose shape could be approximated by multi-faces. Benefit from the Gauss map, the surface Gaussian curvature could be reflected on the 2D spherical surface. Due to the distributional independence of each face through Gauss mapping, we can decide the modality by comparing the distributional character.

In Section 2 we will review the Gauss-Bonnet theorem applying to piecewise surface concisely. Based on it, we will analyze an approximated Gauss mapping method for triangular surface. After that, a mesh filter to eliminate the illogical mapped points is introduced. In Section 3, we will describe the alignment method on Gauss map. Here, the K-means clustering method is used to obtain the same quantity of the respective points, which are utilized to remark the modalities of the models. And we also give a simple solution of the alignment parameters. Evaluation method incorporating corresponding landmark points is introduced in the last section. Some experimental results will be illustrated after that. 


\section{Surface Generation and Its Gauss Map}

\subsection{Organic Polygonal Surface Generation}

The choice of shape representation is the first fundamental decision when designing statistical shape models. The surface meshes representation is one of the most generic methods. As well as it is convenient to general landmarks using mesh resample technique. To get polygonal surface training samples from body CT images, firstly, we should extract the region of interest and represent it by binary voxel data. The mesh is extracted by Marching Cubes Algorithm introduced by Lorensen and Cline [5]. But the smoothness of the surface mesh generated by this algorithm is quite rough. The mesh mean filter is optional for mesh smoothing.

\subsection{Gauss Map of Polygonal Surface}

The Gaussian curvature of a point on a surface is an intrinsic measure of curvature, i.e., its value depends only on how distances are measured on the surface. The GaussBonnet theorem links total curvature of a surface to its topological properties [6,7]. The theorem also has interesting consequences for triangles. It could be accounted as follows:

Suppose $D$ is a simply connected region on the surface, $\partial D$ is a piecewise smooth closed curve, $k_{g}$ is called the geodesic curvature of the curve. Assume $\alpha_{j}$ are the outer angles of the vertices of $\partial D$. Then

$$
\int_{D} K_{p} \mathrm{~d} A+\int_{\partial D} k_{g} \mathrm{~d} s+\sum_{j} \alpha_{j}=2 \pi
$$

If the geodesic curvature is smooth, then

$$
\int_{D} K_{p} \mathrm{~d} A+\sum_{j} \alpha_{j}=2 \pi
$$

The integral operation will be replaced by the accumulated sum operation, when we consider the piecewise polygonal surface.

Figure 1(a) shows a polygonal cell with three faces adjacent a vertex $V_{i}$. The number of the faces is not limited by three, we denote it by $s . G_{j}$ represents one point in the unit triangular face $V_{i} V_{i+1} V_{i+2}$, and the norm $n_{j}$ joints this triangular face at it. The norm $n_{j}$ is given by

$$
n_{j}=\frac{\left(V_{i+1}-V_{i}\right) \wedge\left(V_{i+2}-V_{i}\right)}{\left|\left(V_{i+1}-V_{i}\right) \wedge\left(V_{i+2}-V_{i}\right)\right|}
$$

As the normal direction of each polygonal plane face is special, mapping this unit face to a point on the Gaussian sphere still makes sense. $G_{j}^{\prime}$ is the corresponding point of $G_{j}$ on Gauss map, in Figure 1(b), and it also used to represent the mapping of the face $V_{i} V_{i+1} V_{i+2}$. According to the angular variety of the two vertices $G_{j}, G_{j+1}$,
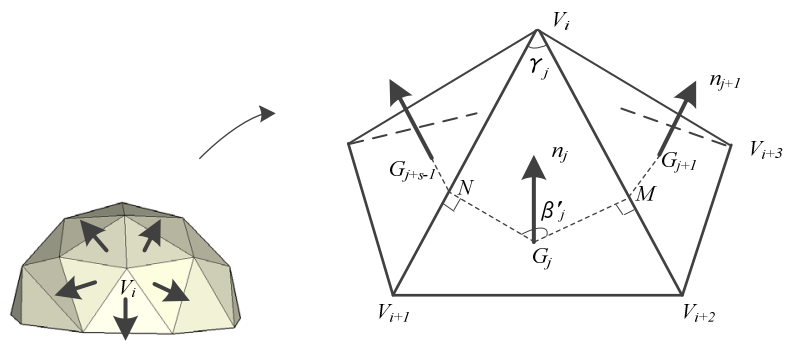

(a)
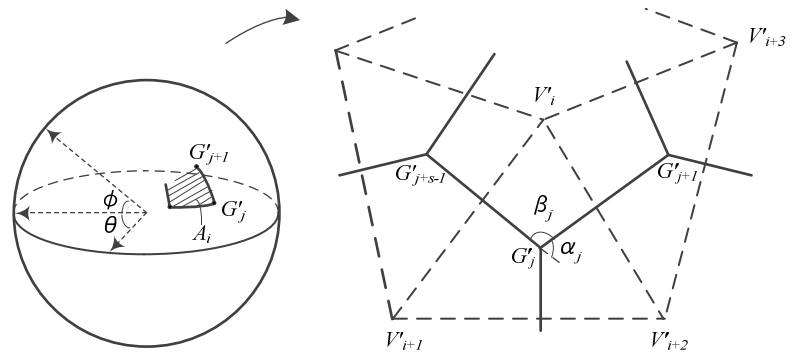

(b)

Figure 1. One polygonal cell around a vertex and its Gauss mapping. (a) The norms of triangular faces adjacent this vertex; (b) One polygonal face unit on Gauss map.

the line segments $G_{j} M, M G_{j+1}$ could be projected to the spherical segment $G_{j}^{\prime} G_{j+1}^{\prime}$. In the same way, mapping of the vertex $V_{i}$ is loaded inside of the simple connection of the region $G_{j}^{\prime} G_{j+1}^{\prime} \cdots G_{j+s-1}^{\prime}$, because the original surface is smooth and continuous.

Support $\alpha_{j}$ are the outer angles of the cell. Because the Gaussian curvature on the Gauss sphere is one everywhere, from the Equation (2), the area of this cell $A_{i}$ could be simplified by

$$
A_{i}=2 \pi-\sum_{j} \alpha_{j}=(2-n) \pi+\sum_{j} \beta_{j}
$$

where $\beta_{j}$ is the intersection angle between two spherical segments. Since spherical segments $G_{j}^{\prime} G_{j+1}^{\prime}$ and $G_{j}^{\prime} G_{j+s-1}^{\prime}$ are the mapping of the segments $G_{j}^{\prime} M G_{j+1}^{\prime}$ and

$G_{j}^{\prime} N G_{j+s-1}^{\prime}$ respectively, if denote the projective angle of $\beta_{j}$ as $\beta_{j}^{\prime}$, it is not difficult to proof that $\beta_{j}^{\prime}$ approaches to $\beta_{j}$.

As well as

$$
\gamma_{i}+\beta_{i}^{\prime}=\pi
$$

then

$$
A_{i}=2 \pi-\sum_{j} \gamma_{j}
$$

The right part of the Equation (5) is the total Gauss curvature at vertex $V_{i}$. So, this equation illustrates that the area of the spherical polygonal cell on Gauss map could be applied to estimate the curvature of the original piecewise polygonal surface. When we minimized the 
area $A_{i}$, the norm of the vertex $V_{i}$ could be approximated by the sum of the norms of the faces around $V_{i}$.

\subsection{Remove the Scattered Mapping Vertices}

At some parts of polygonal surface, such as irregular indentation and the boundary of object, the vigorous change of curvature induces some vertices scattered after gauss mapping. Here, a mesh filter is designed to reduce the influence from that.

Apply the spherical coordinate to express the points on Gauss map. Define $\theta_{i} \in[0, \pi]$ is the inclination angle measured form a fixed zenith direction, and $\varphi_{i} \in[0,2 \pi)$ is the azimuth angle. Then the variance of the spherical coordinates could be formulated by Equation (6), which describes how dispersive the point $V_{i}$ to its neighbors,

$$
\begin{aligned}
\mu_{i} & =E\left[\left(\theta_{i j}-E\left[\theta_{i j}\right]\right)^{2}\right] E\left[\left(\phi_{i j}-E\left[\phi_{i j}\right]\right)^{2}\right] \\
& =\left(E\left[\theta_{i j}{ }^{2}\right]-E\left[\theta_{i j}\right]^{2}\right)\left(E\left[\phi_{i j}{ }^{2}\right]-E\left[\phi_{i j}\right]^{2}\right)
\end{aligned}
$$

where $\theta_{i j}=\theta_{j}-\theta_{i}, \phi_{i j}=\phi_{j}-\phi_{i}$ and $E$ is the mean operator. The "noise point" could be defined as which is bigger than threshold.

\section{Alignment on Gauss Map}

\subsection{Distribution of Local Surface Feature on Gauss Map}

On the Gauss map, we can facilitate to obtain the estimation of geometric attributes of surface such as curvature distribution, uniform continuity and smoothness. To the same graphic pattern, this estimation has the similarity in some sense.

In Figure 2(a), we demonstrate mapping a 2D shape to Gauss sphere. The cambered surface patches which have continuous curvature are mapped continuous regions on Gauss map spherical surface. The plane part $\mathrm{P}_{0} \mathrm{P}_{1}$ is concentrated into a point $n_{0}$. The patch $\mathrm{P}_{1} \mathrm{P}_{2}$ is convex line segment with a low curvature, the mapping is continuous from $n_{1}$ to $n_{2}$ with a relative dispersive distribution. The concave patch $\mathrm{P}_{2} \mathrm{P}_{3} \mathrm{P}_{0}$ has a bevel near the point $\mathrm{P}_{3}$, is reflected region to $n_{2} n_{3} n_{4} n_{5}$ is seemed separated. Since the discontinuity of curvature among the regions of surface, the mapping points aggregate to some separations. The modalities could be inferred from this "texture of curvature". Figure 2(b) shows a Gauss map of one case of liver polygonal triangular surface. The mapping distribution is distinct.

\subsection{Matching Solution by K-Means Clustering}

Unfold the Gauss map according to the spherical coordi- nates, as Figure 3(a), cluster of the point sets is obvious. The intensive regions correspond to the main faces which are relative flat on the original polygonal surface of organic model. Following it, if find a metric to measure the distances between the main surfaces, the optimi zation of alignment method could be performed.

The cluster method is used to describe the distribution of the point sets. In detail, here we use the K-means clustering algorithm to divide the point sets on 2D Gauss map. The centroid of a cluster, denoted as the representative point, is the average point in the multidimensional space defined by the dimensions. In a sense, it is the center of gravity for the respective cluster. In this method, the distance between two clusters is determined as the difference between centroids. Reference [8] provides a boosting algorithm which uses kd-tree structure. The number of the classifiers is 21 and the initial seed points' positions are set averagely.

The solution of the rigid parameters solution is based on Berthold K.P. Horn [9]. The author provides a method to decompose the effects from the translating, scale and

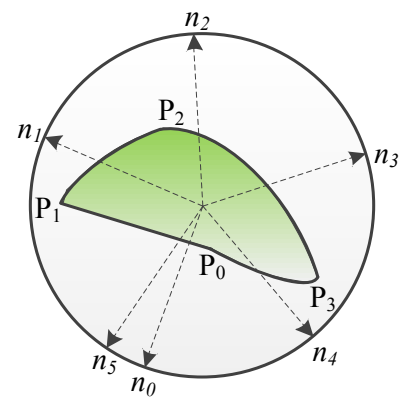

(a)

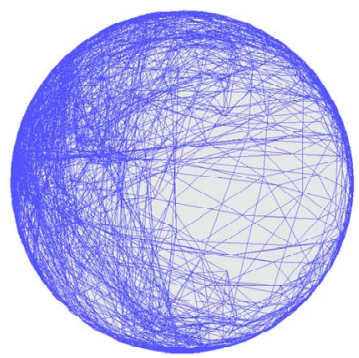

(b)
Figure 2. Gaussian sphere. (a) Gauss mapping of 2D model; (b) Gauss mapping of liver triangular surface.

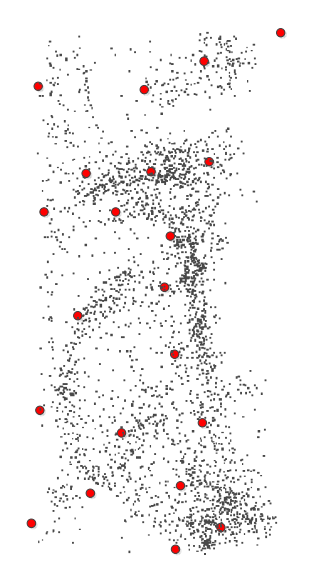

(a)

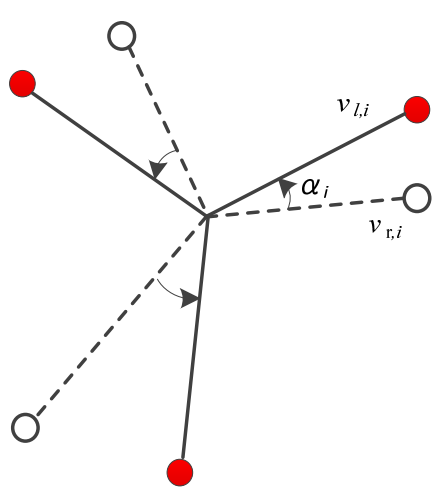

(b)
Figure 3. Alignment method illustration. (a) Representative points obtained by k-means method. Shown on unfolded Gauss map; (b) Rotation parameter optimization. 
rotation. It is a coarse alignment method and could give a simple solution when all the points are coplanar. In that case, it has been inferred that to require the translation minimize, just align the centers of gravity of two point sets. Referring to the scale factor, actually it needn't be considered, for all surface maps are in the unit sphere. The remaining task about the rotation is the solution of a least squares problem in a plane, as Figure 3(b). The vector $v_{l, i}$ points from one representative point to the centroid on Gauss map of referent model, Corresponding it $v_{r, i}$ is on the sample model. $\alpha_{i}$ represents the angle between these two vectors pair. The solution of the rotation amounts to minimize the

$$
\sum_{i}\left\|v_{l, i}-v_{r, i}\right\|^{2}
$$

The optimized deviation angle $\theta$ could be calculated by

$$
\sin \theta= \pm \frac{S}{\sqrt{S^{2}+C^{2}}}
$$

where $C=\sum_{i}\left(v_{l, i} \cdot v_{r, i}\right), S=\sum_{i}\left(v_{l, i} \cdot v_{r, i}\right)$.

\section{Experiments Results}

\subsection{Similarity Criterion}

Due to the intense variety of organ shapes, seeking for corresponding points seems to be a difficult task. There is ever lack of reliable measures to quantify model quality yet. In this study, the selection of the label points depending on the anatomical structure drew an easy way of implement. There are two steps: the position matching that reconciling the center of models and regular the size of the samples by mean radius of the whole vertices. Then we choose 20 points, we call them label points, on the polygonal surface. Evaluate the results by measure the Euclidean distances sum of corresponding points.

\subsection{Verification Experiments}

To verify the alignment extent by proposed method, we aligned one set of left lung training shapes, which generated by one lung polygonal surface model but the modalities were changed through rotation in $3 \mathrm{D}$ space. Using the same model is convenient to the quantitative analysis. Map the vertices to the Gauss map and align the points set using the method in Section 3.2. The results are recorded in Table 1. The rotation volume represents the rotation values of the model along the $\mathrm{x}$ axis, $\mathrm{y}$ axis and $\mathrm{z}$ axis respectively. The Pre-Alignment is the original mean distances of the label points and the PostAlignment corresponds the distances after alignment.

\subsection{Fitness Results}

Alignment experiments were performed on training samples of left lung field and liver field. To evaluate the results of alignment, we compared the Euclidean distances sum of corresponding label points between training samples and reference model. The improvement of distances sum of the label points is applied to evaluate the availability of proposed method. 4 cases triangular polygonal surface samples of left lung field and 3 cases on liver were implied. The rotation column records the angle adjustment, discussed in Section 3.2. As well as the improvement rate is shown in Tables 2-3. The Pre-Alignment is the original mean distances of label points. The Post-Alignment is the corresponding distances after alignment.

\subsection{Discussion}

According to the alignment evaluation, the relative position of the training samples to the reference model is improved. The average improvement of Euclidean distances of label points is $1.3 \%$ on left lung field alignment experiment results and $0.23 \%$ on liver field.

In [10], the author points out that in fact few registration papers attempt to follow up on the use of the Registration. Many cases the registration problem is just satisfied the visualization requirement. In this research, the purpose of the alignment is that force unique points on the surface of training samples to their corresponding points on referenced model. So the precision rate could be improved with the alignment rate improving.

In this paper, we employed the plane Euclidean distance as the evaluate argument of the alignment method.

Table 1. Confirmation results on rotated one case of lung trainning sample.

\begin{tabular}{cccc}
\hline Case & Offset $(x, y, z)$ & Pre-Alignment & Post-Alignment \\
\hline 1 & $0,0,0.0175$ & 0.0381 & 0.0346 \\
2 & $0,0.0175,0$ & 0.1956 & 0.0191 \\
3 & $0.0175,0,0$ & 0.0810 & 0.0799 \\
4 & $0.0349,0.0349,0$ & 0.4380 & 0.4424 \\
\hline
\end{tabular}

Table 2. Fitness results on left lung trainning samples.

\begin{tabular}{ccccc}
\hline Case & Rotation & Pre-Alignment & Post-Alignment & Improvement \\
\hline 1 & 0.0291 & 0.3214 & 0.3090 & $3.9 \%$ \\
2 & 0.0707 & 0.3395 & 0.3381 & $0.4 \%$ \\
3 & 0.0356 & 0.2503 & 0.2481 & $0.9 \%$ \\
4 & 0.0551 & 0.3340 & 0.3342 & $-0 \%$ \\
\hline
\end{tabular}


Table 3. Fitness results on liver trainning samples.

\begin{tabular}{ccccc}
\hline Case & Rotation & Pre-Alignment & Post-Alignment & Improvement \\
\hline 1 & 0.0385 & 0.3565 & 0.3543 & $0.6 \%$ \\
2 & 0.0405 & 0.6078 & 0.6012 & $1.1 \%$ \\
3 & -0.0012 & 0.4422 & 0.4467 & $-1 \%$ \\
\hline
\end{tabular}

But on the Gauss map, the distance metric is spherical. Its approximate evaluation by plane distance would induce the measurement error. Especially when the oriental deviate of the training sample is large, as the case 4 in Table 1 showed. Another sensible factor to the alignment accuracy is that the representative points generated by K-means algorithm could not reflect the distribution density of the mapping points. A better way to solve this problem is using classifier method to assign the weight to each representative point.

\section{Conclusions}

In this paper, we proposed a feature based alignment method to match a set of training shapes for SSM model building. Considering the shape correspondence processing of the model building, we proposed a feature based method and transform the 3D object alignment problem into 2D Gaussian spherical space. Here, we introduced the piecewise Gauss map theoretical foundation and gave an approximated solution for triangular polygonal surface. As well, we proposed using representative points to reflect the modality of the point sets on Gauss map. While the K-means based clustering method was employed to obtain these representative points. The experimental results on the left lung and liver training samples showed the availability of the proposed method. However, in theory, this method is not elaborate yet, because using Gauss map cannot describe the character of surface reliably all the time.

As the future works, we will improve the surface parameterization method by conformal geometry theory. Reference [11] provides a previous works in this area. It is undoubtedly a convictive way for the model alignment and landmarks registration. As well, we will use spherecal distance to replace the plane Euclidean distance when metric the points on Gauss map. At last but not the least, the classifier algorithm is expected to get a better description of the distributional regularity of surface points.

\section{Acknowledgements}

We would like to thank our colleagues and friends at the Kyushu Institute of Technology for their research basis on medical image processing. Especially thank interna- tional exchange student Eloi Duchaussoy for always having an open ear for many smart ideas to me. Thank T. Heimann at Medical and Biological Infomatics at the German Cancer Research Center. Without his kindly help about the programming of triangular mesh structure, we couldn't complete this research on time. He is very accommodating and intelligent.

\section{References}

[1] A. T. Heimann and H. Meinzer, "Statistical Shape Models for 3D Medical Image Segmentation: A review," Medical Image Analysis, Vol. 13, No. 4, 2009, pp. 543563. doi:10.1016/i.media.2009.05.004

[2] T. F. Cootes and C. J. Taylor, "Statistical Models of Appearance for Medical Image Analysis and Computer Vision," SPIE Medical Imaging, Vol. 4322, No. 3, 2001, pp. 236-248.

[3] J. B. A. Maintz and M. A. Viergever, "A Survey of Medical Image Registration," Medical Image Analysis, Vol. 2, No. 1, 1998, pp. 1-36. doi:10.1016/S1361-8415(98)80001-7

[4] M. A. Audette, F. P. Ferrie and T. M. Peters, "An Algorithmic Overview of Surface Registration Techniques for Medical Imaging," Medical Image Analysis, Vol. 4, No. 3, 2000, pp. 201-217. doi:10.1016/S1361-8415(00)00014-1

[5] W. E. Lorensen and H. E. Cline, "Marching Cubes: A High Resolution 3D Surface Construction Algorithm," ACM SIGGRAPH Computer Graphics, Vol. 21, No. 4, 1987, pp.163-169. doi:10.1145/37402.37422

[6] D. S. Meek and D. J. Walton, "On Surface Normal and Gaussian Curvature Approximations Given Data Sampled from Smooth Surface," Computer Aided Geometric Design, Vol. 17, No. 6, 2000, pp. 521-543. doi:10.1016/S0167-8396(00)00006-6

[7] X. F. D. Gu and S.-T. Yau, "Computational Conformal Geometry," Higher Education Press, Beijing, 2008, pp. 90-95.

[8] T. Kanungo, N. S. Netanyahu and A. Y. Wu, "An Efficient K-Means Clustering Algorithm: Analysis and Implementation," IEEE Transactions on Pattern Analysis and Machine Intelligence, Vol. 24, No. 7, 2002, pp. 881892. doi:10.1109/TPAMI.2002.1017616

[9] B. K. P. Horn, "Closed-Form Solution of Absolute Orientation Using Unit Quaternions," Journal of the Optical Society of America A, Vol. 4, No. 4, 1987, pp. 629-642. doi:10.1364/JOSAA.4.000629

[10] T. Zrimec, S. Busayarat and P. Wilson, "A 3D Model of the Human Lung," Medical Image Computing and Computer-Assisted Intervention, Vol. 3217, 2004, pp. 1074 1075. doi:10.1007/978-3-540-30136-3 143

[11] X. Gu, Y. Wang, T. F. Chan, P. M. Thompson and S. Yau, "Genus Zero Surface Conformal Mapping and Its Application to Brain Surface Mapping," IEEE Transactions on Medical Imaging, Vol. 23, No. 8, 2004, pp. 949-958. doi:10.1109/TMI.2004.831226 